\title{
Basıncın ve Sıcaklığın Dış Elektrik Alan Altındaki Kübik Kuantum Noktasının Üçüncü Harmonik Üretimi Üzerine Etkisi
}

\author{
The Effect of Pressure and Temperature on the Third Harmonic Generation of Cubical \\ Quantum dot under External Electric Field
}

\author{
Muharrem KIRAK* \\ Bozok Üniversitesi, Eğitim Fakültesi, Temel Ĕ̆itim Bölümü, 66100, Yozgat
}

• Geliş tarihi / Received: 09.03.2018 • Düzeltilerek geliş tarihi / Received in revised form: 08.06.2018 • Kabul tarihi / Accepted: 22.06 .2018

\begin{abstract}
$\ddot{\mathbf{O z}}$
Düşük boyutlu sistemler, yük taşıyıcılarının nano ölçekte sınırlandırıldığı yapılardır. Yük taşıyıcı hareketinin üç boyutta sınırlandırıldığı yapılar kuantum noktalar olarak bilinir ve bu yapılar moleküler biyoloji uygulamalarında, tıbbi görüntülemede, bilgi depolamada, optik ve iletişim gibi aygıt uygulamalarında önemli bir rol oynar. Bu çalışmada, dış elektrik alan altındaki kübik kuantum noktasının üçüncü harmonik üretimi üzerine basıncın ve sıcaklığın etkisi teorik olarak incelenmiştir. Sayısal hesaplamalar etkin kütle yaklaşımı altında yapılmıştır. Yapının taban durumu ve uyarılmış durumların enerji özdeğerleri hesaplanmış ve elde edilen bu değerler optik özelliğin hesaplanmasında kullanılmıştır. Aynı zamanda, kübik kuantum nokta boyutu ve durulma oranı etkisi de incelendi. Sonuçlar basınç ve sıcaklığın üçüncü harmonik üretimi üzerine büyük bir etkisinin olduğunu göstermektedir.
\end{abstract}

Anahtar kelimeler: Basınç, Kübik kuantum nokta, Sıcaklık, Üçüncü harmonik üretimi

\begin{abstract}
Low dimensional systems are structures in which the charge carriers are confined in nanoscale. The structures that the movements of charge carriers are restricted in three dimensions are known as quantum dot and these structures play important role in application of molecular biology, medical imaging, storage of data, application devices such as optics and communication. In this study, the effects of pressure and temperature on the third harmonic generation of cubic quantım dot under external electric field theoretically have been investigated. Numerical calculations have been done within the effective-mass approximation. The energy eigenvalues of the ground state and excited states of the structure have been calculated and the obtained these values have been used in calculation of optical properties. Also, the effects of size of cubical quantum dot and the relaxation rate have been investigated. Results show that the pressure and temperature has a great influence on third harmonic generation.
\end{abstract}

Keywords: Pressure, Cubical quantum dot, Temperature, Third harmonic generation

* Muharrem KIRAK; muharrem.kirak@ bozok.edu.tr; Tel: (0354) 2421025 (dâhili: 7809); orcid.org/0000-0003-3208-2242 


\section{Giriş}

Kuantum kuyu, kuantum tel ve kuantum nokta gibi kuantum nanoyapılar, külçe (bulk) malzemelere göre oldukça farklı fiziksel özelliklere sahiptir. Bundan dolayı, bu yapılar hem mikroelektronik hem de optoelektronikte oldukça ilgi çekici yapılardır. Kuantum nanoyapılar, bir alt tabaka üzerine atomik ölçekte veya daha büyük kalınlıkta tabakaların büyütülmesiyle oluşan yapılardır. Diğer yapılara göre daha üstün özelliklere sahip olan kuantum noktaları, taşıyıcıların üç boyutta sınırlandırıldığı yapılardır ve yapay atom olarak da adlandırılırlar (Maksym vd., 1990; Kouwenhoven vd., 1998). Günümüzde, modern büyütme teknikleri ile küresel, silindirik ve kübik gibi farklı formlarda kuantum noktaların üretilmesi mümkün hale gelmiştir. Kuantum nokta tabanlı yapılar yariiletken lazerler (Kirstaedter vd., 1996), güneş pilleri (Aeberhard vd., 2008), lazer diyotlar (Darabi vd., 2006) ve k1zılötesi fotodedektörler (Jiang vd., 1999) gibi cihaz uygulamaları için oldukça benzersiz özellikler sergilemektedir. Son zamanlarda kuantum nokta yapıların elektronik ve optik özellikleri yoğun bir şekilde çalışılmıştır.

Elektrik alan, manyetik alan, hidrostatik basınç ve sıcaklık gibi dış faktörlerin kuantum nokta yapıların fiziksel özellikleri üzerine etkisi gerek teorik gerekse deneysel çalışmalarda önemli bir yer teşkil etmektedir. Dış elektrik alan altındaki kübik GaAs/AlAs kuantum noktasının elektron alt -bant enerjisi Dane ve ark. (2007) tarafindan teorik olarak incelenmiştir. Bu çalışmada elektron alt-bant enerjisinin elektrik alan ile azaldığını gösterilmiştir. Spector ve Lee (2007) kübik kuantum noktasında elektrik alanın elektronların taban durumu ve birinci uyarılmış durum üzerine etkisini hesaplamıştır. Sonlu ve sonsuz sınırlama potansiyeli altında kuantum kutusunda bantlar arası optik geçişler için soğurma katsayısı ve kırılma indisi değişimi Karabulut ve ark. (2005, 2006) tarafindan incelenmiştir. Diğer taraftan, hidrostatik basınç altında kübik kuantum noktasının optik özellikleri Khordad ve ark. (2011) tarafından çalışılmıştır. Uygulanan dış elektrik alan altındaki kübik kuantum noktası için safsızlık konumlarının lineer ve lineer olmayan optik özellikler üzerine etkisi Kirak ve Yilmaz (2015) tarafindan incelenmiştir. Bu çalışmada elde edilen sonuçlar safsızlık konumlarının ve elektrik alanın sistemin optik karakteristikleri üzerine büyük bir etkisinin olduğunu göstermiştir.

Kuantum nanoyapıların en ilginç optik özelliklerden birisi de üçüncü harmonik üretimidir. Üçüncü harmonik üretimi çok hızlı optik anahtarlar, elektro-optik modülatör, biyogörüntüleme sensörler, tek elektron transistörler, fotodedektörler ve kızı̈ötesi lazerler gibi pek çok uygulamaya sahip olan en temel lineer olmayan optik süreçlerden biridir (Sibilia vd., 2008). Üçüncü harmonik üretimi lineer olmayan malzeme üzerine gönderilen fotonların frekansının üç kat1 büyüklügünde frekansa sahip bir radyasyon üretimine neden olur. Üçüncü harmonik üretimi hem simetrik hem de asimetrik potansiyele sahip yapılarda gözlenebilir (Li, 1996). Üçüncü harmonik üretimi içeren lineer olmayan alınganlık kuantum kuyusu (Zaluzny vd., 1996; Yildirim vd., 2006 ), kuantum teli (Wang, 2005; Wang vd., 2008) ve kuantum noktasi (Karabulut vd., 2009; Shao vd., 2010; Yu vd., 2011) için çeşitli araştırmacılar tarafından incelenmiştir. Kübik kuantum noktasında üçüncü harmonik üretimi Zhang ve ark. (2009) tarafindan çalışılmıştır. Bununla birlikte, literatüre bakıldığında kübik kuantum noktasında hidrostatik basıncın ve sicaklığın üçüncü harmonik üretimi üzerine etkisi incelenmemiştir. $\mathrm{Bu}$ alandaki yapilacak olan incelemeler hem teorik olarak hem de pratik uygulamalar açısından önemli olacaktır.

Bu çalışmada, basıncın ve sıcaklığın dış elektrik alan altındaki kübik GaAs kuantum noktasında üçüncü harmonik üretimi üzerine etkisi incelenmiştir. Makalenin geri kalan kısmı şu şekilde düzenlenmiştir. Çalışmanın teorik altyapıs1 özet olarak Bölüm 2'de verilmiştir. Nümerik sonuçlar Bölüm 3'de verilmiș ve tartışılmıștır. Son olarak, Bölüm 4'te sonuç kısmı verilmiştir.

\section{Teori}

Bu bölümde, dış elektrik alan, hidrostatik basınç ve sıcaklık etkisinde kübik kuantum noktasını dikkate alacağız. Etkin kütle yaklaşımı ile birlikte, dış elektrik alan varlığında üç boyutta sistemin Hamiltonyeni aşağıdaki gibi verilir.

$H=-\frac{\hbar^{2}}{2 m^{*}(P, T)} \nabla^{2}+|e| F z+V_{c}(x, y, z)$.

Burada, $\hbar$ indirgenmiş Planck sabiti, $e$ elektronun yükü, $F$ elektrik alan büyüklüğ̈̈, $P$ hidrostatik basınç ve $T$ sıcaklıktır. $m^{*}(P, T)$, basınca ve sıcaklığa bağlı elektronun etkin kütlesidir. $V_{c}(x, y, z)$, sinırlandırıcı potansiyeldir ve kuantum noktası içinde sıfir diğer durumlarda sonsuz alınmıştır. 
Sınır şartlarına bağlı olarak (1) denkleminin çözümü

$\Psi_{n_{x} n_{y} n_{z}=\phi_{n_{x}}(x) \phi_{n_{y}}(y) \phi_{n_{z}}(z)}$

şeklinde olacaktır. Burada $n_{x}, n_{y}$ ve $n_{z}$ kuantum sayılarıdır. z- yönünde uygulanan elektrik alan varlığında gerekli çözümler yapıldığında dalga fonksiyonu,

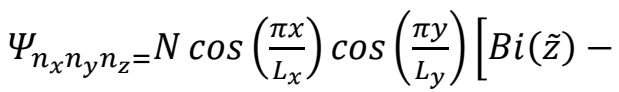

$$
\begin{aligned}
& \left.\frac{B i\left(\xi_{L}\right)}{A i\left(\xi_{L}\right)} A i(\tilde{z})\right]
\end{aligned}
$$

olarak elde edilir. Burada $N$ normalizasyon katsayıs1 ve $A i$ ve $B i$ sirası ile birinci ve ikinci tip Airy fonksiyonlarıdır. Kübik kuantum noktasının kenar uzunlukları $L_{x}, L_{y}$ ve $L_{z}$ olarak tanımlanmıştır. (3) numaralı denklemde $x<$ $\left|L_{x} / 2\right|, y<\left|L_{y} / 2\right|, \quad z<\left|L_{z} / 2\right|, \quad \xi_{L}=\tilde{z}(z=$ $\left.-L_{x} / 2\right)$ ve $\xi_{R}=\tilde{z}\left(z=L_{x} / 2\right)$ şeklindedir.

Aynı zamanda

$\tilde{z}=\left(\frac{2 m^{*}(P, T) e F}{\hbar^{2}}\right)^{-2 / 3}\left[\frac{2 m^{*}(P, T)}{\hbar^{2}}(|e| F z-E)+\left(\frac{n_{x} \pi x}{L_{x}}\right)^{2}+\left(\frac{n_{y} \pi y}{L_{y}}\right)^{2}\right]$

şeklindedir. Burada $E$ enerji ifadesidir. Basınç ve sıcaklığa bağlı etkin kütle aşağıdaki gibi tanımlanır (Welber vd., 1975) :

$m^{*}(P, T)=m_{0}\left(1+E_{P}^{\Gamma}\left(\frac{2}{E_{g}^{\Gamma}(P, T)}+\frac{1}{E_{g}^{\Gamma}(P, T)+\Delta_{S O}}\right)\right)^{-1}$.

Burada, $m_{0}$ serbest elektronun kütlesini, $E_{P}^{\Gamma}$ momentum matris elemanı ile ilgili enerjiyi, $\Delta_{S O}$ GaAs için valans bandının spin-yörünge yarılmasını göstermektedir. $E_{g}^{\Gamma}(P, T)$, hidrostatik basınç ve sıcaklık bağlı olarak $\Gamma$ noktasında enerji aralığı değişimi olarak tanımlanır ve şu şekilde verilir (Duque vd., 2012):
$E_{g}^{\Gamma}(P, T)=E_{g}^{\Gamma}(0)+b P-\frac{\alpha T^{2}}{T+\beta}$.

Burada, $E_{g}^{\Gamma}(0)$ sıfır basınçta $(P=0)$ enerji aralığı $b$ lineer basınç katsayısı, $\alpha$ ve $\beta$ Varshni parametreleridir. Ayrıca, GaAs için basınç ve sıcaklığa bağlı dielektrik sabiti şu şekilde verilir (Samara, 1983):

$\varepsilon(P, T)= \begin{cases}12.74 \exp \left(-16.7 \times 10^{-3} P\right) \times \exp \left(9.4 \times 10^{-5}(T-75.6)\right), & T<200 \\ 13.18 \exp \left(-17.3 \times 10^{-3} P\right) \times \exp \left(20.4 \times 10^{-5}(T-300)\right), & T \geq 200\end{cases}$

Kübik noktası boyutlarının basınç ile değişimi (Sali vd., 2014)

$L(P)=L_{0} C(P), \quad C(P)=\left[1-3\left(S_{11}+2 S_{12}\right) P\right]^{1 / 3}$,

ile verilir.

Burada, $L_{0}$ kübik noktasının orijinal uzunluğu, $S_{11}$ ve $S_{21}$ uyum sabitleridir. $T=300 \mathrm{~K}$ için GaAs parametre değerleri Tablo 1'de verilmiştir.

$3 w$ frekanslı üçüncü harmonik üretimi, ikinci harmonik üretiminin aksine inversiyon simetriye sahip kristallerde bile mümkündür. Sisteme monokromatik elektromanyetik alanın $E(t)=$ $E_{0} \cos w t=\tilde{E} e^{i w t}+\tilde{E} e^{-i w t} \quad$ şeklinde uygulandığını varsayalım. Yoğunluk-matris yaklaşımı ve iteratif süreç kullanarak (Ahn vd., 1987; Rosencher vd., 1991), üçüncü harmonik üretimi katsayısı aşağıdaki gibi elde edilir (Wang ve Guo, 2001). 
Tablo 1: $T=300 \mathrm{~K}$ için GaAs parametre değerleri

\begin{tabular}{|l|l|l|l|}
\hline Parametre & Değer & Parametre & Değer \\
\hline$E_{g}^{\Gamma}(0)(\mathrm{meV})$ & 1519 & $\beta(\mathrm{K})$ & 204 \\
\hline$\Delta_{S O}(\mathrm{meV})$ & 341 & $b(\mathrm{meV} / \mathrm{GPa})$ & 107.3 \\
\hline$E_{g}^{\Gamma}(P, T)(\mathrm{meV})$ & 7510 & $S_{11}\left(\times 10^{-2}\left(\mathrm{GPa}^{-1}\right)\right.$ & 1.16 \\
\hline$\alpha(\mathrm{meV} / \mathrm{K})$ & 0.5405 & $S_{21}\left(\times 10^{-2}\left(\mathrm{GPa}^{-1}\right)\right.$ & 0.37 \\
\hline
\end{tabular}

$$
\begin{aligned}
& \chi_{3 w}^{(3)}=\frac{e^{4}}{\varepsilon_{0}} \sum_{i} \sum_{k} \frac{M_{k i}}{3 \hbar w-\hbar w_{k i}-i \hbar \Gamma_{k i}} \sum_{l} \sum_{j}\left[\frac{M_{i l} M_{l j} M_{j k}\left(\sigma_{k}-\sigma_{j}\right)}{\left(2 \hbar w-\hbar w_{k l}-i \hbar \Gamma_{k l}\right)\left(\hbar w-\hbar w_{k j}-i \hbar \Gamma_{k j}\right)}-\frac{M_{i l} M_{l j} M_{j k}\left(\sigma_{j}-\sigma_{l}\right)}{\left(2 \hbar w-\hbar w_{k l}-i \hbar \Gamma_{k l}\right)\left(\hbar w-\hbar w_{j l}-i \hbar \Gamma_{j l}\right)}-\right. \\
& \left.\frac{M_{i j} M_{j l} M_{l k}\left(\sigma_{l}-\sigma_{j}\right)}{\left(2 \hbar w-\hbar w_{l i}-i \hbar \Gamma_{l i}\right)\left(\hbar w-\hbar w_{l j}-i \hbar \Gamma_{l j}\right)}+\frac{M_{i j} M_{j l} M_{l k}\left(\sigma_{k}-\sigma_{i}\right)}{\left(2 \hbar w-\hbar w_{l i}-i \hbar \Gamma_{l i}\right)\left(\hbar w-\hbar w_{j i}-i \hbar \Gamma_{j i}\right)}\right]
\end{aligned}
$$

Burada $i, k, l, j=0,1,2,3$. İki foton rezonans koşulu göz önüne alındığında (9) denklemi şu şekilde basitleştirilebilir.

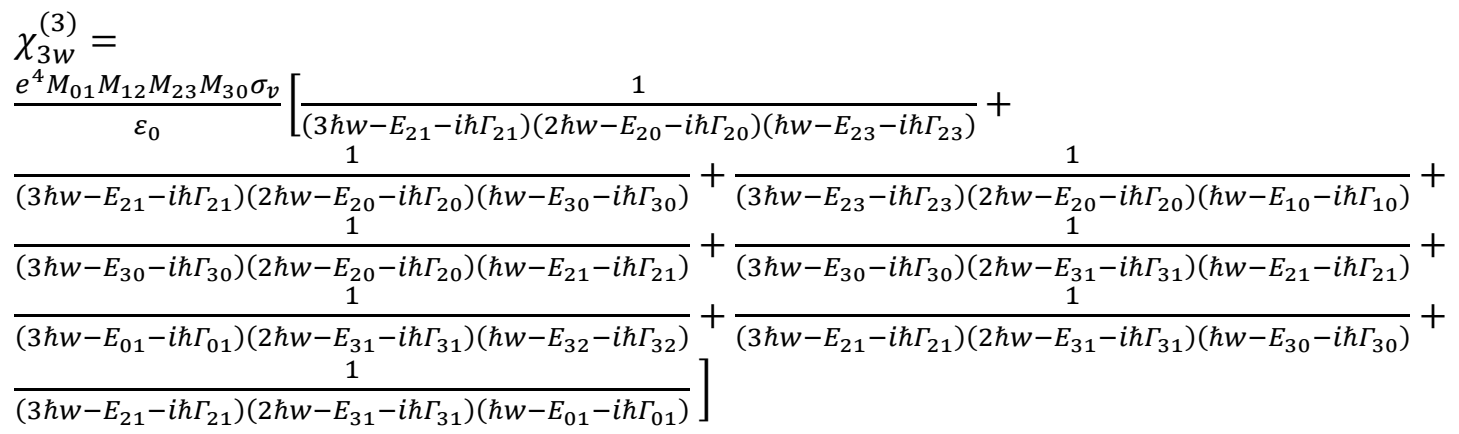

Burada, $\sigma_{V}$ elektron yoğunluğu, $e$ elektronun yükü, $\varepsilon_{0}$ boşluğun geçirgenlik katsayısı, $\Gamma_{i j}, i$ ve $j$ durumları için durulma oranı, $\hbar w$ foton enerjisidir. $M_{i j}, i$ ve $j$ durumları arasında dipol geçiş elemanıdır ve $M_{i j}=$ $e\left\langle\psi_{i}|z| \psi_{j}\right\rangle$ olarak tanımlanır. $E_{i j}=E_{i}-E_{j}$ iki elektronik seviye arasında enerji farkıdır ve $M_{01} M_{12} M_{23} M_{30}$ geometrik faktördür. Polarize olmuş elektromanyetik ışıma z- doğrultusunda seçilmiştir.

\section{Nümerik Sonuçlar ve Tartış̧ma}

Bu bölümde; sıcaklık, basınç, uzaysal sınırlama ve durulma oranının dış elektrik alandaki kübik kuantum noktasının üçüncü harmonik üretimi üzerine etkileri tartışlacaktır. Nümerik hesaplamalar boyunca atomik birimler $\left(\hbar=m_{0}=\right.$ $e=1$ ) kullanıldı. $\mathrm{Bu}$ birimlerde etkin $\mathrm{Bohr}$ yarıçap1 $a^{*}=\hbar^{2} \varepsilon(P, T) / m^{*}(P, T) e^{2} \quad$ ve etkin Rydberg enerjisi $R^{*}=m^{*}(P, T) e^{4} / \hbar^{2} \varepsilon(P, T)^{2}$ olarak tanımlandı. Diğer parametreler $\sigma_{v}=$ $5 \times 10^{24} \mathrm{~m}^{-3}, \quad \hbar \Gamma_{10}=\hbar \Gamma_{21}=\hbar \Gamma_{32}=\hbar \Gamma \mathrm{meV}$, $\hbar \Gamma_{20}=\hbar \Gamma_{31}=\hbar \Gamma / 2 \mathrm{meV}$ ve $\hbar \Gamma_{30}=\hbar \Gamma / 3 \mathrm{meV}$ olarak alındi.

Şekil 1(a)'da üç farklı sıcaklık değeri için $(T=4 K, T=200 \mathrm{~K}$ ve $T=400 K)$ gönderilen foton enerjisinin bir fonksiyonu olarak üçüncü harmonik üretimi değişimi verilmiştir. Bu grafik $L=10 \mathrm{~nm}, P=0$ ve $F=65 \mathrm{kV} / \mathrm{cm}$ değerleri için çizilmiş̧tir. Grafik incelendiğinde $E_{20} / 2$,
$E_{30} / 3$ ve $E_{31} / 2$ değerlerinde olmak üzere üç farklı rezonans pik genliği görülmektedir. Diğer rezonans pik genlikleri baskın olmadığı için oldukça küçük olup grafik üzerinde gösterilmemiştir. Şekil 1(a)'dan görüldüğü gibi sicaklık arttıkça pik genliği daha düşük foton enerji değerlerine kaymaktadır $\mathrm{ki}$ bu durum kırmızıya doğru adlandırılır. Bunun nedeni ise sıcaklık arttıç̧a etkin kütlenin azalması ve dielektrik sabitinin artmasıdır. Böylece etkin Rydberg enerjisi azalmaktadır. Bununla birlikte, sicaklık arttıkça $\chi_{3 w}^{(3)}$ büyüklüğü sıcaklıkla artmaktadır. Bu artışın fiziksel nedeni sıcaklık ile etkin Bohr yarıçapı ve dipol matris elemanlarının çarpımının, $M_{01} M_{12} M_{23} M_{30}$, artmasıdır. $\chi_{3 w}^{(3)}$ büyüklüğü dipol matris elemanlar çarpımı ile orantılıdır. Şekil 1(b)'den açıkça görüldüğü gibi dipol matris elemanlar çarpımı sıcaklıkla artmaktadır. Bu şekil, kübik kuantum noktasında üçüncü harmonik üretiminin sıcaklığa hassas bir şekilde bağlı olduğunu göstermektedir. 

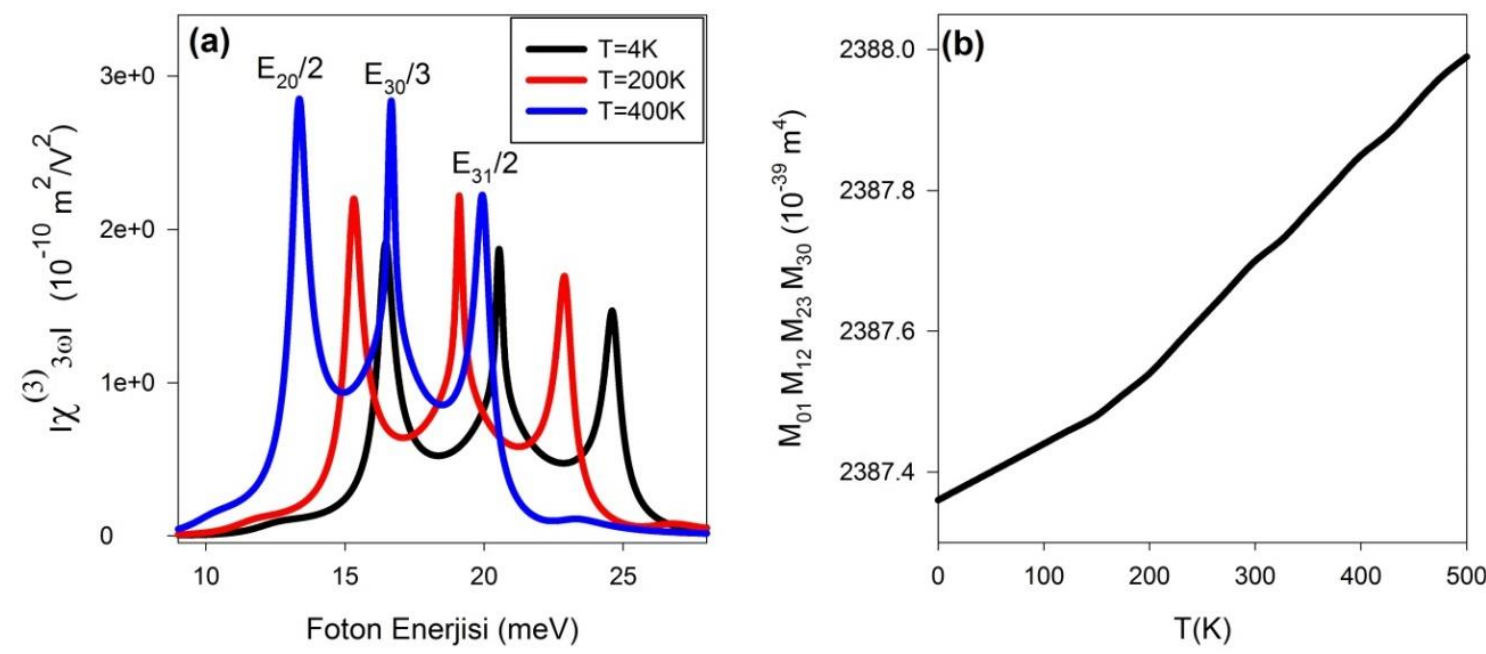

Şekil 1. $L=10 \mathrm{~nm}, P=0, F=65 \mathrm{kV} / \mathrm{cm}$ ve $\hbar \Gamma=1.0 \mathrm{meV}$ değerleri için a) Üçüncü harmonik üretiminin foton enerjisinin fonksiyonu olarak üç farklı sıcaklık değeri için değişimi b) Dipol matris elemanları çarpımının sıcaklıkla değişimi.

Üçüncü harmonik üretiminin basınca bağl1lığı $(P=0,1,2 \mathrm{GPa}$ değerleri için) $L=10 \mathrm{~nm}$, $T=200 K$ ve $F=65 \mathrm{kV} / \mathrm{cm}$ değerleri için Şekil 2'de verilmiştir. Şekilden görüldüğü gibi basınç arttıkça üçüncü harmonik üretiminin pik genliği daha yüksek enerji değerlerine kaymaktadır, yani maviye kaydığı gözlenmektedir.

Basıncin artmasıyla birlikte etkin kütle artmakta, dielektrik sabiti azalmakta ve böylece etkin Rydberg enerjisi artmaktadır. Ayrıca, üçüncü harmonik üretiminin büyüklüğü basınçla birlikte azalmaktadır. Bunun nedeni ise basıncin artmas1 ile etkin Bohr yarıçapının ve dipol matris elemanları çarpımının azalmasıdır (Bkz. Şekil 2(b)).

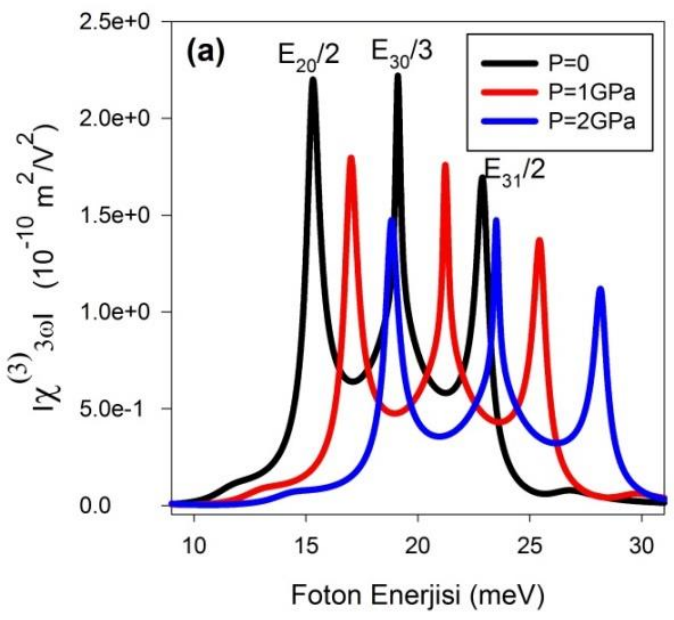

Şekil 3(a) üç farklı kübik kuantum noktası uzunluğu için foton enerjisinin fonksiyonu olarak üçüncü harmonik üretiminin değişimini göstermektedir. Grafik $F=65 \mathrm{kV} / \mathrm{cm}, T=$ $200 \mathrm{~K}, P=1 \mathrm{GPa}$ ve $\hbar \Gamma=1.0 \mathrm{meV}$ değerleri için çizilmiştir. Şekil 3(a)'dan görüldüğü gibi kübik kuantum noktası uzunluğunun artması ile üçüncü harmonik üretimi pik genliği artmaktadır. Şekil 3(b)'den açıkça görüldüğü gibi kübik kuantum noktasının uzunluğu arttıkça dipol matris elemanları çarpımı artmaktadır. Bununla birlikte kübik kuantum noktası uzunluğu arttıkça pik genlikleri kırmızıya kayma davranışı sergilemektedir. Üçüncü harmonik üretiminin rezonans pik genlik değerleri Tablo 2' de verilmiştir.

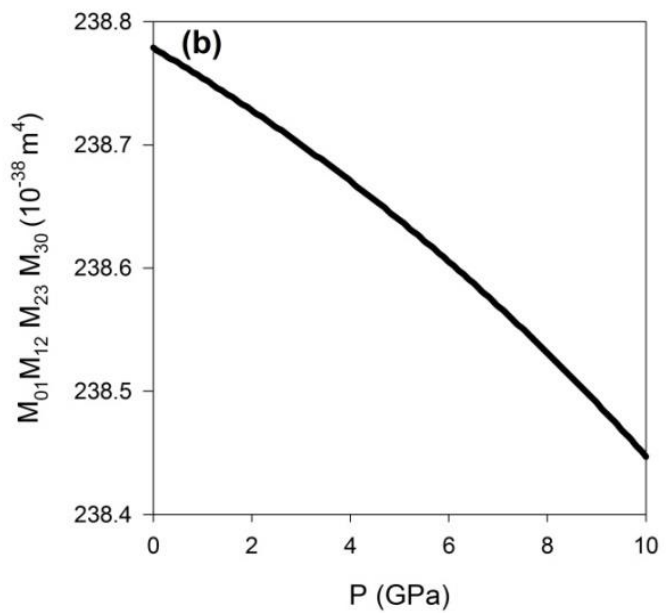

Şekil 2. $L=10 \mathrm{~nm}, T=200 \mathrm{~K}, F=65 \mathrm{kV} / \mathrm{cm}$ ve $\hbar \Gamma=1.0 \mathrm{meV}$ değerleri için a) Üçüncü harmonik üretiminin foton enerjisinin bir fonksiyonu olarak üç farklı basınç değeri için değişimi b) Dipol matris elemanları çarpımının basınçla değişimi 

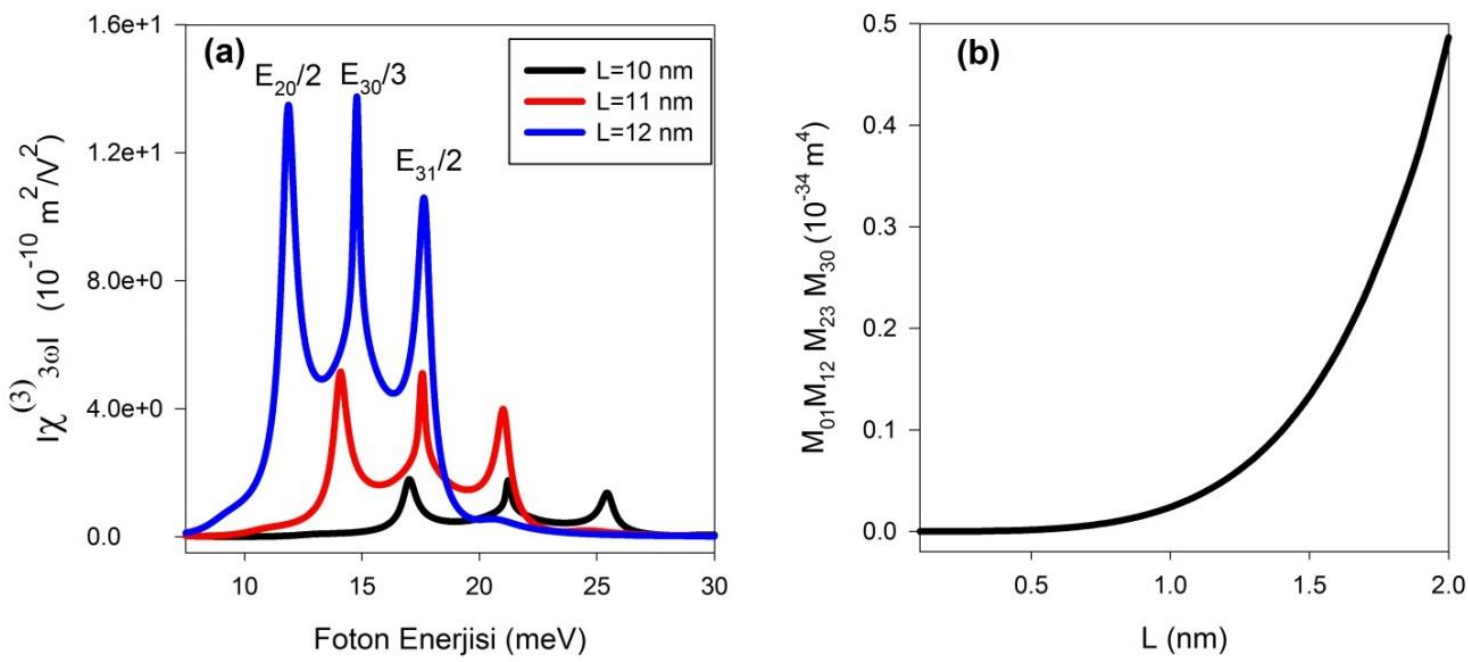

Şekil 3. $F=65 \mathrm{kV} / \mathrm{cm}, T=200 \mathrm{~K}, P=1 \mathrm{GPa}$ ve $\hbar \Gamma=1.0 \mathrm{meV}$ değerleri için a) Üçüncü harmonik üretiminin foton enerjisinin bir fonksiyonu olarak üç farklı kübik kuantum noktası uzunluğu değeri için değişimi b) Dipol matris elemanları çarpımının kübik kuantum noktası uzunluğu ile değişımi

Tablo 2: Şekil 1, Şekil 2 ve Şekil 3 için rezonans pik genlik değerleri

\begin{tabular}{|lllll|}
\multicolumn{1}{l}{} & & $\boldsymbol{E}_{\mathbf{2 0}} / \mathbf{2}$ & $\boldsymbol{E}_{\mathbf{3 0}} / \mathbf{3}$ & $\boldsymbol{E}_{\mathbf{3 1}} / \mathbf{2}$ \\
\hline \multirow{3}{*}{ Şekil 1. için } & $T=4 K$ & $16.5 \mathrm{meV}$ & $20.5 \mathrm{meV}$ & $24.6 \mathrm{meV}$ \\
& $T=200 \mathrm{~K}$ & $15.3 \mathrm{meV}$ & $19.1 \mathrm{meV}$ & $22.9 \mathrm{meV}$ \\
& $T=400 \mathrm{~K}$ & $13.4 \mathrm{meV}$ & $16.7 \mathrm{meV}$ & $19.9 \mathrm{meV}$ \\
\hline \multirow{3}{*}{ Şekil 2. için } & $P=0$ & $15.3 \mathrm{meV}$ & $19.1 \mathrm{meV}$ & $22.9 \mathrm{meV}$ \\
& $P=1 G P a$ & $17.0 \mathrm{meV}$ & $21.2 \mathrm{meV}$ & $25.4 \mathrm{meV}$ \\
& $P=2 G P a$ & $18.8 \mathrm{meV}$ & $23.5 \mathrm{meV}$ & $28.1 \mathrm{meV}$ \\
\hline \multirow{3}{*}{ Şekil 3. için } & $L=10 \mathrm{~nm}$ & $17.0 \mathrm{meV}$ & $21.2 \mathrm{meV}$ & $25.4 \mathrm{meV}$ \\
& $L=11 \mathrm{~nm}$ & $14.1 \mathrm{meV}$ & $17.6 \mathrm{meV}$ & $20.9 \mathrm{meV}$ \\
& $L=12 \mathrm{~nm}$ & $11.9 \mathrm{meV}$ & $14.8 \mathrm{meV}$ & $17.6 \mathrm{meV}$ \\
\hline
\end{tabular}

Son olarak, farklı durulma oranları için foton enerjisinin bir fonksiyonu olarak üçüncü harmonik üretimi Şekil 4'te çizilmiştir. Bu grafikte $F=65 \mathrm{kV} / \mathrm{cm}, T=200 \mathrm{~K}, P=1 \mathrm{GPa}$ ve $L=10 \mathrm{~nm}$ değerleri alınmıştır. Açıkça görüldüğü gibi $\quad 17.0 \mathrm{meV}, \quad 21.2 \mathrm{meV}$ ve $25.4 \mathrm{meV}$ değerlerinde maksimum pik genlik değerleri gözlenmektedir. Durulma oran1 arttıkça üçüncü harmonik pik genliği değerinde azalma meydana gelmektedir. Durulma oranı, durulma zamanının tersi olup spektrumun yarı genişliğiyle orantılıdır. Durulma zamanın arttıkça spektrum daha dar hale gelir. Dolayısıyla durulma oran1 arttıkça spektrum genişleyecek ve bu da pik genlik değerinin azalmasına neden olacaktır. Küçük durulma oranları için daha keskin pik genlik değerleri olmaktadır. Bununla birlikte, durulma oranındaki değişme rezonans piklerin konumunu değiştirmemektedir. Bunun nedeni elektronik seviyelerinin enerjileri durulma oranından bağımsız olmasından kaynaklanmaktadir.

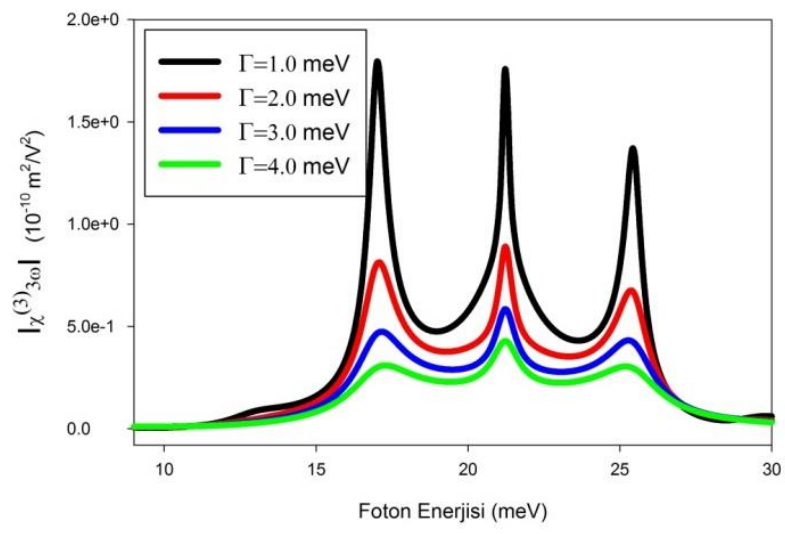

Şekil 4. $F=65 \mathrm{kV} / \mathrm{cm}, T=200 \mathrm{~K}, P=1 G P a$ ve $L=10 \mathrm{~nm}$ değerleri için üçüncü harmonik üretiminin foton enerjisinin bir fonksiyonu olarak dört farklı durulma oranı değeri için değişimi 


\section{Sonuç}

Dış elektrik alan altındaki kübik GaAs kuantum noktası için üçüncü harmonik üretimi üzerine hidrostatik basınç, sicaklık, kübik kuantum noktası uzunluğu ve durulma oranı etkileri incelenmiştir. Üçüncü harmonik üretiminin bu faktörlere oldukça duyarlı olduğu bulunmuştur. Nümerik hesaplamalar göstermektedir ki sıcaklık (basınç) arttıkça üçüncü harmonik üretimi büyüklüğü artmakta (azalmakta) ve bu optik özellik kırmızıya (maviye) kayma davranışı sergilemektedir. Kübik kuantum noktası boyutları arttıkça üçüncü harmonik değeri büyümekte ve rezonans pik genlikleri daha düşük foton enerjilerine kaymaktadır. Durulma oranı ise sadece üçüncü harmonik üretimi büyüklügüne etki etmekte, durulma oranı arttıkça büyüklük azalmaktadır. Ancak rezonans pik değerleri değişmemektedir. Elde edilen veriler 1şı̆̆ında basınç ve sıcaklık değişimi ile üçüncü harmonik üretimi istenilen şekilde ayarlanabilir. $\mathrm{Bu}$ çalışmanın ileri ki tarihlerde yapılacak olan teorik ve deneysel araştırmalara katk1 sağlayacağ 1 düşünülmektedir. Basınç ve sıcaklığın dış elektrik alan altındaki kübik kuantum noktasında üçüncü harmonik üretimi üzerine etkisi en iyi bilgimiz dâhilinde daha önce incelenmemiştir. Yapılan bu çalışma teorik olarak literatüre önemli bir katk1 sağlayacak ve kuantum nokta yapıların özelliklerinin daha iyi anlaşılmasına yardımcı olacaktır. Ayrıca, bu çalışma ile elde edilen sonuçlar kuantum nokta temelli aygit uygulamaları için erişilecek bir veri ortaya koyacaktır.

\section{Kaynaklar}

Ahn, D. ve Chuang, S. L. 1987. Calculation of Linear and Nonlinear. Intersubband Optical Absorptions ina Quantum Well Mode1 with an Applied Electric Field. IEEE Journal of Quantum Electronics, 23, 2196-2204.

Dane, C., Akbas, H., Talip, N. ve Kasapoglu, K., 2007. Effect of spatial electric field on the sub-band energy in a cubic GaAs/AlAs quantum dot. Physica E: Low-dimensional Systems and Nanostructures, 39, 95-98.

Duque, C. A., Mora-Ramos, M. E., Kasapoglu, E., Sari, H. ve Sokmen, I. 2012. Combined effects of intense laser field and applied electric field on exciton states in GaAs quantum wells: Transition from the single to double quantum well. Physica Status Solidi (b), 249, 118-127.
Karabulut, I., Unlu, S. ve Safak, H. 2005. Calculation of the changes in the absorption and refractive index for intersubband optical transitions in a quantum box. Physica Status Solidi (b), 242, 2902-2909.

Karabulut, I. ve Baskoutas, S.. 2009. Second and Third Harmonic Generation Susceptibilities of Spherical Quantum Dots: Effects of Impurities, Electric Field and Size. Journal of Computational and Theoretical Nanoscience, 6, 153-156.

Khordad, R., Rezaei, G., Vaseghi, B., Taghizadeh, F. ve Kenary H. A. 2011. Study of optical properties in a cubic quantum dot. Optical and Quantum Electronics, 42, 587-600.

Kirak, M. ve Yilmaz, S. 2015. Impurity position effects on the linear and nonlinear optical properties of the cubic quantum dot under an external electric field. Journal of Physics D: Applied Physics, 48, 325301-325301.

Kouwenhoven, L. ve Marcus, C. 1998. Quantum dots. PhysicsWorld, 11, 35-39.

Li, E. H. 1996. Interdiffusion as a means of fabricating parabolic quantum wells for the enhancement of the nonlinear third-order susceptibility by triple resonance. Applied Physics Letters, 69, 460.

Maksym, P.A. ve Chakrabotry, T. 1990. Quantum dots in a magnetic field: role of electronelectron

interactions. American Physical Society, 65, 108111.

Rosencher, E. ve Bois, Ph. 1991. Model system for optical nonlinearities: Asymmetric quantum wells. Physical Review B, 44, 11315 .

Sali, A. ve Satori, H. 2014. The combined effect of pressure and temperature on the impurity binding energy in a cubic quantum dot using the FEM simulation. Superlattices and Microstructures, 69, 38-52.

Samara, G. A. 1983. Temperature and pressure dependences of the dielectric constants of semiconductors. Physical Review B, 27, 3494.

Shao, S., Guo, K. X., Zhang, Z. H., Li, N. ve Peng, C. 2010. Studies on the third- 
harmonic generations in cylindrical quantum dots with an applied electric field. Superlattices and Microstructures, 48, 541549.

Sibilia, C., Benson, T., Marciniak, M., Szoplik, T. 2008. Photonic Crystals: Physics and Technology, 1st edn, Springer, Milano.

Spector, H. N. ve Lee, J., (2007). Stark effect in the optical absorption in cubical quantum boxes. Physica B: Condensed Matter, 393, 94-99.

Unlu, S., Karabulut, I. ve Safak, H. 2006. Linear and nonlinear intersubband optical absorption coefficients and refractive index changes in a quantum box with finite confining potential. Physica E: Lowdimensional Systems and Nanostructures, 33, 319-324.

Wang, G. ve Guo, K. X. 2001. Excitonic effects on the third-harmonic generation in parabolic quantum dots. Journal of Physics: Condensed Matter,13, 8197-8206.

Wang, G. 2005. Third-harmonic generation in cylindrical parabolic quantum wires with an applied electric field. Physical Review B, $72,155329$.
Wang, G. ve Guo, Q. 2008. Third-harmonic generation in cylindrical parabolic quantum wires with static magnetic fields. Physica B: Condensed Matter, 403, 37-43

Welber, B., Cardona, M., Kim, C. K. ve Rodriquez, S. 1975. Dependence of the direct energy gap of GaAs on hydrostatic pressure. Physical Review B, 12, 5729.

Yildirim, H. ve Tomak, M. 2006. Third-harmonic generation in a quantum well with adjustable asymmetry under an electric field. Physica Status Solidi (b), 243, 40574063.

Yu, Y. B. ve Wang, H. J. 2011. Third-harmonic generation in two-dimensional pseudo-dot system with an applied magnetic field. Superlattices and Microstructures, 50, 252260.

Zaluzny, M. ve Bondarenko, V. 1996. Influence of the depolarization effect on thirdharmonic generation in quantum wells. J. Appl. Phys. 79, 6750-6754.

Zhang, Z. H., Guo, K. X., Chen, B., Wang, R. Z. ve Kang, M. W. 2009. Third-harmonic generation in cubical quantum dots. Superlattices and Microstructures, 46, 672678. 